\title{
Digital Microfluidics: Techniques, Their Applications and Advantages
}

\author{
Sarvesh Sukhatme ${ }^{1 *}$ and Ajay Agarwal ${ }^{2}$
}

${ }^{1}$ Undergraduate student at the Birla Institute of Technology and Science, Pilani (BITS-Pilani), India

${ }^{2}$ CSIR-Central Electronics Engineering Research Institute, Pilani, Rajasthan 333031, India

\begin{abstract}
Digital Microfluidics is a recent advent of the miniaturization boom that can be witnessed over the couple of decades. The conventional techniques developed for continuous microfluidics cannot necessarily be used directly for application to digital microfluidics. This paper presents a brief overview of the techniques used for performing different operations in Digital Microfluidics namely Electro-wetting, Dielectrophoresis (DEP), Opto-electro-wetting (OEW), Thermo-capillary effect, Surface Acoustic Waves (SAW), etc.
\end{abstract}

Keywords: Digital microfluidics; Electrohydrodynamics; Electrowetting on dielectric, Optoelectrowetting; Dielectrophoresis

\section{Introduction}

Digital microfluidics uses discrete samples of liquids or rather drops/droplets for performing the functions as those of continuous microfluidics discretely, analogous to the difference between Analog and Digital Microfluidics[1,2]. As in continuous microfluidics streams of liquids in micro-channels with flow rates in $\mu \mathrm{L}$ are used for analysis while in Digital Microfluidics individual droplets are used instead of a continuous flow of liquid. The devices used are somewhat similar to that of conventional microfluidics, except for the additional circuits for creation and transport of droplets. In some cases additional hardware is required to perform more operations like cutting, merging etc. The physics governing microfluidics [3] is applicable to digital microfluidics and has been well verified. But there are some rare exceptions for droplets as the reduction in size poses difficulties like rapid evaporation, electric breakdown, wetting etc. Use of micropumps and other micro-scale mechanical elements is reduced as electronic actuation is preferred due to miniaturization of electrodes and the ease of integration into a single device which decreases effective size [4]. Silicon and its other derivatives like PDMS (Poly-dimethylsiloxane) are the preferred material for fabrication of digital microfluidic devices.

Digital Microfluidics offers several advantages over continuous domain microfluidics; hence the field is rapidly progressing and encompassing more and more researchers towards this concept. Due to handling of individual droplets the sample volume is further reduced to nano-liter or pico-liter. As demonstrated by microfluidics, lower the reagent volume the faster are the results [5]. Hence individual droplets give even faster results than continuous flows. Also the amount of reagent required in chemical and biological assays which can be difficult to obtain and also economically infeasible can be used in very small amounts thus reducing wastage and economizing the problem. The scaling of volumes used also reduces the device size thus creating several opportunities for portable devices which can be easily used. Perhaps, the most significant advantage of digital microfluidics is that several test circuits can be created and operated in parallel on the same device thus speeding up the process. A continuous flow from the source or a reservoir can be established and droplet formation circuit will form several droplets directly fed to parallel assays on the same device, performing various tests on the same device. Monolithic design techniques can enable patterning of several testing modules on the device.

But it is easier said than done. There are several difficulties involved in handling of micro-droplets. As mentioned earlier, drops evaporate more rapidly than continuous flows hence extreme care has to be taken in reduction of heat transfer. Transportation of droplets is another tedious task requiring accurate creation of device geometries without slightest imperfections. Surface patterning of electrodes etc. requires silicon derivatives and conventional fabrication techniques like Lithography, etching, laser micro-machining etc. which are not simple and not economic. Extreme care has to be taken in the choice of material for every function it has to perform as regards with the liquid in concern.

The applications of Digital Microfluidics are wide encompassing various disciplines like Biology, Chemistry, Chemical Engineering etc. and are still expanding. The most notable of them include portable assays like DNA, PCR and Proteomics. Proteomics is the study of different proteins which often requires creation of numerous samples and having them react with different reagents for in depth-study. This is often lengthy and tedious. With Digital Microfluidics, many samples can be created and analyzed in parallel on the same chip thus effectively saving time and improving efficiency. Introduction of Optofluidics has revolutionized Biomedical Imaging. As the device is on a micro-scale it can capture images of high resolution as well as magnification by integrating the device with imaging electronic circuits. This can be a splendid component of monitoring devices.

Researchers have overcome some of these difficulties and successfully demonstrated devices, implementing digital microfluidics that suggests the significant potential of this concept. Various techniques utilized in Digital Microfluidic devices are discussed in following sections.

\section{Electro wetting}

Surface phenomenon is quite dominant when dealing at the microscale. Inertial and other phenomenon like gravitation are negligible while considering the flow. Electro-wetting utilizes the concept of

*Corresponding author: Sarvesh Sukhatme, Undergraduate student at the Birla Institute of Technology and Science, Pilani (BITS-Pilani), India, E-mail: sarveshsukhatme@gmail.com

Received June 22, 2012; Accepted August 27, 2012; Published August 30, 2012

Citation: Sukhatme S, Agarwal A (2012) Digital Microfluidics: Techniques, Their Applications and Advantages. J Bioengineer \& Biomedical Sci S8:001. doi:10.4172/2155-9538.S8-001

Copyright: (c) 2012 Sukhatme S, et al. This is an open-access article distributed under the terms of the Creative Commons Attribution License, which permits unrestricted use, distribution, and reproduction in any medium, provided the original author and source are credited. 
surface tension property of a liquid. When a normal liquid is placed on a surface it wets the surface due to the surface tension acting on the molecules at the membrane of the liquid-air interface and the forces of attraction between the liquid and the surface. The similar case is with droplets, when placed on a surface it reforms into a shape which minimizes its surface energy which usually is spherical. The contact angle is the angle formed by the droplet interface meets the surface. In electro-wetting, the contact angle is changed by applying an external charge or potential on the liquid (Figure 1). By asymmetrically changing the contact angle on both sides, droplet actuation can be achieved. The physics of electro-wetting has been briefly discussed by Mugele and Baret [6]. Droplet dynamics have also been studied [7]

Changing the contact angle asymmetrically to create a pressure gradient along the droplet which results in actuation has been demonstrated [8]. Electro-wetting for formation of droplets from an on chip reservoir has been done [9]. Capacitance measurement was used as a feedback mechanism for controlling the droplet size. Electrowetting requires high amount of potentials, generally exceeding 10 volts to cause actuation of droplets. Such high voltages might cause droplets to breakdown or evaporate. Hence, a subtle modification is used instead of direct Electro-wetting, called Electro-Wetting-onDielectric (EWOD). The dielectric layer used above the electrodes in the device doesn't allow the complete potential to drop across the droplet. Another point worth noting is that the voltages used are AC and not DC as the capacitance of the droplet blocks the entire potential, for DC voltages, and no actuation can be achieved.

A typical EWOD device consists of a dielectric layer on which the droplet is placed; a layer of closely arranged or packed electrodes is placed on the chip. The voltage is applied to these electrodes. The arrangement of electrodes is such that no two electrodes which are in contact with the droplet are simultaneously ON; else the pressure difference that results will be similar on both sides. Two types of EWOD devices have been developed: Open and Closed. Open devices use only a single substrate is used while in closed two plates are used and the droplet is sandwiched between them .Both the plates have a hydrophobic surface beneath which the other layers follow. Also in the closed system, the top plate is always grounded while in single plate devices, the ground and electrodes are on the same substrate. This review is confined to close plate systems, however many open plate models have also been demonstrated [10].

Electro-wetting has been used not only for creation of droplets but also for cutting, merging and transporting [11]. The droplets are pulled by applying voltage to the electrodes in front of the reservoir and switching them consecutively in the forward direction resulting

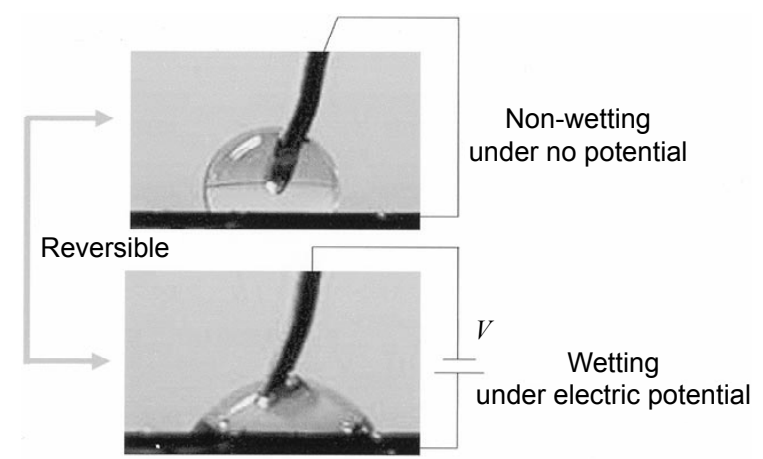

Figure 1: A demonstration of electrowetting. Reprinted with permission of [11].
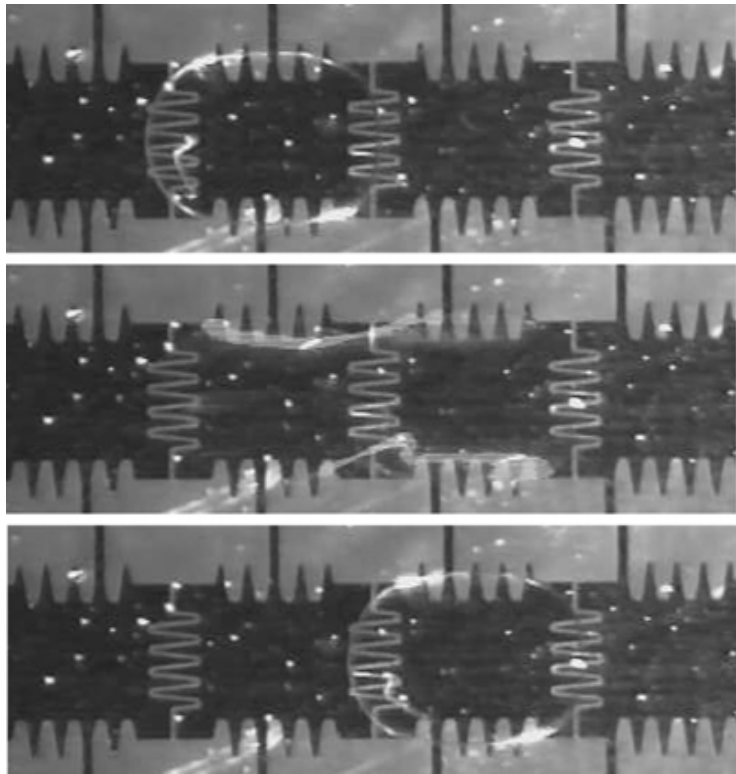

Figure 2: Transportation of a droplet by initially applying voltage to the electrode underneath the droplet in first frame, and then subsequently to the electrodes on the right in the second and third frames. Reprinted with permission from [16]

in pulling of a single meniscus from the reservoir. As it progresses at a certain instant the hydrophobic layers cause the droplet to break out from the reservoir. Transporting, merging was similarly achieved by alternatingly applying voltages to the electrode array on the bottom plate (Figure 2) . For merging, several electrode arrays have to be operated simultaneously, in order to bring the two or more droplets in contact. In depth numerical modeling of electro-wetting transport is given by [12-14]. Electro-wetting has been performed in air [15], silicone oil [16]. Feedback mechanism for control of droplets with complete electronic control for EWOD has been done [17].

Electro-wetting remains the most exploited and studied technique for implementation in Digital Microfluidics. Due to its simple principle and reduction in mechanical elements it is the popular choice as a technique. The electrode arrays required are created using the same fabrication techniques as those for Integrated circuits. Hence, not much innovation is required and the available literature is abundant. These methods are well proven so chances of errors are very small. As AC voltages are used, transmission and application is relatively simpler. Electro-wetting depends on surface tension, which is the determining factor hence external agents like surfactants are rendered useless. By altering the frequency and magnitude high speeds of transport can be achieved. Due to simplicity, Electro-wetting can be easily integrated with Lab-On-Chip systems without affecting the size and functioning while providing thorough output screening. Polymerase Chain reaction (PCR) has been accomplished on digital microfluidic device using EWOD [18]. Electro-wetting provides several advantages over conventional techniques like $\mathrm{T}$-junction, flow focusing geometries for droplet formation. Additional hardware need not be used without sacrificing accuracy. The patterning of intricate structures like $\mathrm{T}$ is a tedious procedure. Altering the flow rates of co-flowing streams requires repeated calculations and labor for changing the droplet size, while in EWOD feedback measurement assures accuracy and reproducibility, Voltage frequency and magnitude can be easily altered to change the size.

There are some considerations which need to be addressed before 
electro-wetting can be implemented. Type of liquids can undergo electro-wetting [19], contact angle hysteresis and saturation, the saturation of the contact angle above a certain voltage level [20], etc. are main considerations. If the angle saturation is quite low, then the liquid droplet cannot be actuated. Only conductive liquids can show electro-wetting, so for non-conducting liquids alternatives have to be found. These difficulties have been overcome and actuation with contact angles as high as 120 degrees has been achieved.

\section{Optofluidics}

Opto-fluidics can be termed as the combination of Microfluidics and Optics. What this essentially conveys is that all the operations performed in a microfluidic platform need not be controlled only mechanically or electronically but by using light. It may not be necessary that only light is the principal part but it may indirectly be responsible for actuation. The first Optical manipulation methods were Optical tweezers [21] and Optical Vortex [22]. The basic principle was inducing an electric field by the presence of Electric field of light falling on the target. Instantly, we can make out that this principle works well only at very small scales in the domain of microfluidics. The induced electric field and the radiation pressure result in the formation of a trap center which can be controlled by varying intensity of light used. By using holographic optical tweezers, arrays of optical vortices can be induced, which serve as very good trap points. They can similarly be tailored for separation of a range of particles from a given mixture [23]. These devices used some micro-mechanical elements in conjunction with optics to perform the set of functions. The same can be done by using electronic elements like microelectrode arrays, which is known as Opto-electro fluidics [24]. Instead of conventional Optical Tweezers, Electronic tweezers are formed using microelectrode arrays beneath a photo-conductive surface. This provides a better control over the device and accuracy, and higher dynamic ranges are possible indirectly induce mechanical and electrical phenomenon.

Another recent and very popular optical manipulation technique introduced for Digital Microfluidics is Opto-Electro-wetting (OEW). The mechanism involved is essentially Electro-wetting but not by alternatingly changing the voltage across several micro-electrodes, but by using Optics [25]. The device consists of a transparent, hydrophobic layer like Indium-Titanium Oxide (ITO) as the top plate so that light can pass through it. The other plate consists of a glass (or silicone) substrate on which a photoconductor like $\mathrm{Si}: \mathrm{H}$ is deposited along with a dielectric. When a potential is applied, there is no actuation of the droplet involved. But when a light beam is incident on the photoconductive surface, this results in the actuation of the droplet.

This can be better visualized by the equivalent electric circuit indicated in (Figure 3) [26]. Here, each component has its resistive

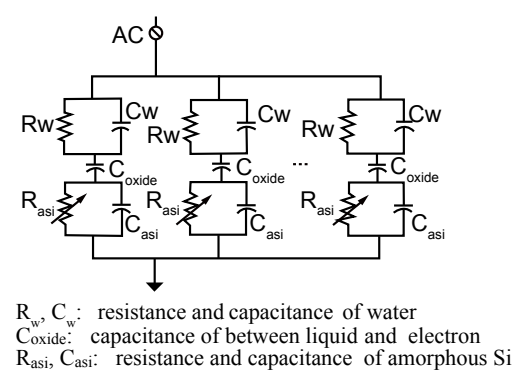

Figure 3: The equivalent circuit for Opto-electrowetting device. Reprinted with permission from [26]. and capacitive elements in series. The only variable parameters are those of the photoconductive material. This is crucial and brilliant as it has to be actuated by light. When there is no light incident on the photoconductor surface, it has a high dark resistance and most of the voltage drops across the photoconductive layer, leaving only a small drop across the droplet. Since the voltage across the drop is negligible, it results in a very small change in the contact angle, which is not enough to cause actuation. When light is incident, the conductivity of the photoconductor $(\mathrm{Si}: \mathrm{H})$ increases and consequently, its resistance decreases. By voltage divider, a significant voltage drops across the droplet and hence there is a large contact angle change that results in the actuation of the droplet. This has been achieved by using a laser beam projected on the microfluidic device. There are several considerations to this principle for application. Only AC voltages have to be used, due to the presence of capacitive elements. The choice of photoconductor depends on its dark and light resistances. For use in an OEW digital microfluidic device, a photoconductor must have a very high dark resistance $\left(\sim 10^{12} \mathrm{Ohms}\right)$ and a relatively very small light resistance. Also, the frequency bands that can be used for the operation of the device have to be considered for specific applications. A novel study of frequency and illumination on the impedance has been done [27]. Open devices have also been used for implementing opto-fluidic devices [28]. The choice of dielectric material also follows the above protocols. By use of Atomic Layer Deposition (ALD) a very high speed of $2 \mathrm{~cm} / \mathrm{s}$ was achieved [29].

While OET's and Optical Vortices are considered as more of separation techniques than actuation techniques, OEW has been used for performing all the fundamental droplet manipulations: Creating, Transporting, Mixing and Cutting/Splitting, parallel manipulation of large number of droplets has also been demonstrated by the use of several light/laser beams or images [30].

Opto-fluidics is considered to be an excellent alternative for Electro-wetting. Manipulation of many droplets provides an edge to the use of OEW devices. With the advent of semiconductor technology, laser diodes with perfect mono-chromatic and accurate intensity are available, which improves the accuracy of actuation. As several arrays of microelectrodes are not required, the fabrication of device is simplified and has resulted in the reduction of hardware. Use of several new engineered photoconductive materials with enhanced performance is other merit of using opto-fluidic devices. Varying the voltages across the electrodes is not required, hence labor is reduced. OET's have been shown to have better accuracy than conventional methods like Optical Vortex and Tweezers. Opto-fluidic devices hold a prime candidacy for use in bio-photonic systems and micro-devices for use in biomedical imaging. Their integration with these devices and easier formation of lenses and other imaging objects holds great promise in the area.

These devices have some limitations as the photoconductive material and laser beams increases the cost of developing the systems. The cost of device further increases with higher specifications. All photoconductive materials are also not usable for digital microfluidic applications and their availability is also feeble.

\section{Dielectrophoresis}

Dielectrophoresis is the polarization of a non-conductive/dielectric liquid in the presence of an electric field [31]. When no electric field is present, the charged particles in the liquid are randomly oriented thus nullifying the net effect. But, in the presence of an electric field the 
particles with appropriate charge are attracted towards the electric field gradient. The net charge always remains conserved i.e. zero.

$$
f_{\mathrm{CM}}\left(\varepsilon_{\mathrm{p}}^{*}, \varepsilon_{\mathrm{m}}^{*}, \omega\right)=\frac{\varepsilon_{\mathrm{p}}^{*}(\omega)-\varepsilon_{\mathrm{m}}^{*}(\omega)}{\varepsilon_{\mathrm{p}}^{*}(\omega)+2 \varepsilon_{\mathrm{m}}^{*}(\omega)}
$$

$\mathrm{f}_{\mathrm{CM}}$ is the dipolar Clausius-Mosotti factor which is the characterizing parameter of a Dielectrophoretic particle.

$\varepsilon_{\mathrm{p}}, \varepsilon_{\mathrm{m}}$ are the complex permittivities of the particle and the medium respectively. In turn, the complex permittivity is given by:

$$
\varepsilon=\varepsilon_{r}-i \frac{\sigma}{\omega}
$$

Where, $\varepsilon_{r}$ is the dielectric constant; $\sigma$ is the conductivity; $\omega$ is the frequency of the applied Electric Field and $i$ is $\sqrt{-1}$.

Interesting to note is that Alternating Fields are used for stable operation in the microfluidic domain. Two types of Dielectrophoresis have been observed: Positive and Negative. In positive DEP, the particles are attracted towards increasing Electric field and in the case of Negative DEP the particles are attracted to regions of weak Electric field.

Dielectrophoresis has been popularized as an effective particle separation technique by Gascoyne et al. [32]. Dielectrophoresis is a good technique for use in microfluidic devices as the principle of operation is simple and hardware required is less. Since it can be applied to non-conductive liquids also, it covers a wide-span of potential application as compared to just electrophoresis. Binary separation for digital microfluidics has been performed [33]. Also, a recent technique for light induced DEP [34] and travelling wave DEP [35] has been performed. The technique can be easily integrated with other microsystems for analysis or measurement purposes. It also provides a great insight into the coulombic interactions at a micro level.

But the use of Dielectrophoresis for actuation purposes remains to be sufficiently explored as it prevails as a separation technique. It requires fabrication of precise electrodes for the generation of electric field to which the particles are attracted. Sometimes it may also require formation of intricate channels for effective separation. It may have certain disadvantages for use for some particles, if their response to the field is not strong enough to be detected. Dielectrophoresis also requires a suitable medium for proper functioning in regard to the Clausius-Mossotti factor etc.

\section{Thermo capillary effect}

As the name suggests, it involves the use of heat as well as capillary affects. Surface Tension has a nominal dependence on temperature. Therefore a change in temperature can be used to create a difference in surface tension that may result in the actuation of a liquid droplet known as the Thermo-capillary effect. Like in Electro-wetting, the temperature gradient has to be non-uniform on the sides of the droplet to result in actual motion. The phenomenon of moving droplets using this effect is termed as Thermo-Capillary Pumping (TCP).

The mathematics governing TCP is given by [36]. Navier Stokes equation:

$$
\rho\left(\frac{\partial v}{\partial t}+v \cdot \nabla v\right)=-\nabla p+\nabla \cdot \mathrm{T}+\mathrm{f}, \rho\left(\frac{\partial v}{2}\right)
$$

where $\mathrm{v}$ is the flow velocity, $\rho$ is the fluid density, $\mathrm{p}$ is the pressure, $\mathrm{T}$ is the stress tensor, and $\mathbf{f}$ represents body forces (per unit volume) acting on the fluid and $\nabla$ is the del operator

$$
\Delta P=\frac{8 \mu L Q}{\pi r^{4}}
$$

Also, Poiseuille's flow can be approximated for most devices:

Where $\Delta \mathrm{P}$ is the pressure drop, $\mathrm{L}$ is the length of pipe, $\mu$ is the dynamic viscosity, $\mathrm{Q}$ is the volumetric flow rate and ' $r$ ' is the radius of the pipe.

The device has a heating element, a flow channel and a temperature sensing element. The sensing elements generally used are Resistance Temperature Detectors (RTDs). Thermo-capillary manipulation has also been done using holographic beam light [37].

As with electronic control, the TCP devices do not require any moving parts. Depending on the applications, the heater elements might not be required as the temperature gradient might be inherent. They are relatively easier to construct. But it has its disadvantages. The contact angle hysteresis observed is more than in case of Electrowetting and also depends on the liquid to be used. A threshold level of temperature difference is required for actuation of the droplets, as it increases the contact angle hysteresis increases [38]. Accurate temperature generation and detection is required which raises the cost of device.

\section{Surface acoustic waves}

The concept of Surface Acoustic Waves (SAW) was first introduced by Lord Rayleigh in 1885. The acoustic waves produced are sometimes referred as Rayleigh waves. The first demonstration of Surface Acoustic Waves for microfluidics was given by Shia et al. [39]. These waves propagate at the speed of sound. They are created on a piezoelectric substrate by applying a high frequency source to the Interdigitated (IDT) electrodes introduced earlier.

The IDT electrodes have a comb like structure to which the Radio frequency source is applied. IDT's can be made of suitable materials like gold which have high modulus of elasticity to create the required surface waves. The IDT's are placed on the piezoelectric surface. The oscillations of the electrodes induce a stress in the material and due to this a wave is propagated. The wave has two components: Parallel and Perpendicular which enables the entire chip area to be utilized [39].

After fluid drops are placed/created on the chip, the waves created by IDT's strike the droplet and cause actuation of the droplet. Due to the viscosity of drops the resulting intensity transferred to the drop decreases along the direction of propagation. This creates a pressure difference along the droplet that results in actuation. The frequency of RF oscillations used depends on the contact angle of liquid droplet to be actuated. Above this specific threshold, very high speeds of droplet transport can be achieved.

Conventional operations of droplets have been performed using SAW principle (Figure 4). Apart from these, PCR [40] and cell based sorting [41] have also been demonstrated. SAW is a good technique for use in fast, parallel operated devices. But the device fabrication requires sophisticated and intricate details. The material for IDT and piezoelectric substrates are liable to raise the cost of microfluidic devices. Depending on the droplet, the chip area may also vary. 


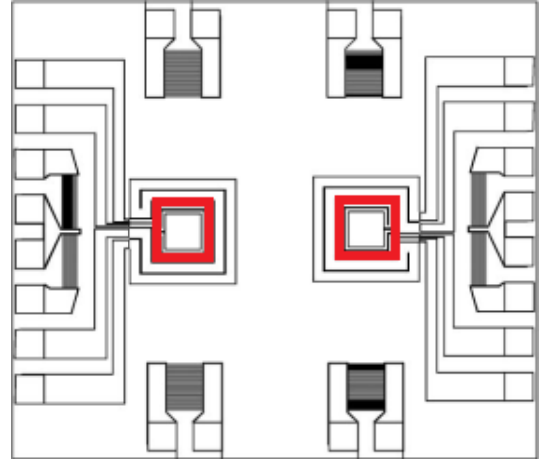

Figure 4: A SAW based digital microfluidic chip. Black rectangles are IDTs, Red rectangles are the two heaters in the center surrounded by temperature sensors and heating wires. Reprinted with permission from [41].

\section{Discussion}

The most common and popular techniques employed in Digital Microfluidic devices have been elucidated. The usage of a particular method depends on the specific function the device is to be used for. Each technique has its own advantages and disadvantages as stated above, but certain performance indices may be developed for narrowing down the choices. The performance indices which will be the benchmark in this paper are: operations that can be performed, ease of device fabrication and economy.

The operations that can be performed in a device are namely generation of droplets, transportation, mixing, cutting and other actuation techniques. Electro-wetting, Opto-Electro-wetting are the techniques that can be used even for generation of droplets while the others can only actuate the droplets, the exception being dielectrophoresis which is more often used only as a separation technique. The efficiency of operating for each technique remains debatable as there are no particular calculations that can prove the least power consuming one. As all the techniques discussed rely on electronic principles rather than mechanical ones they are more preferred over the conventional ones. Electric field is the only major requirement for Electro-wetting, Dielectrophoresis and SAW's while OEW requires an external laser source for operation. TCP requires creation of a temperature gradient by various methods. It is however significant to note that the voltages required for any movement are quite high. Hence, as regard to this index Electro-wetting and OEW are preferable as a wider range of operations can be performed on the same device while for specific purpose of separation DEP is the popular choice.

Device fabrication is an important aspect in the development of any micro-device. The device geometries and the processes performed depend on the function to be carried out by the device. Also the choice of the method used, determine the usability of the device. Since we are functioning on the micro-scale, the fabrication techniques used are usually same as those for manufacturing integrated circuits like lithography, etching etc. Almost all the techniques discussed in the review use the same methods for fabrication but the level of detail and perfection coupled with some extra non-conventional materials used for specific purpose are the parameters that can be used as a basis for efficiency calculation. Electro-wetting and OEW require special patterning of micro-electrodes beneath the substrate for actuation. The general process has been described earlier. As the scaling factor increases, the electrode size has to reduce but the accelerating voltages increase which amounts to a trade-off on these techniques as to how larger voltages can be transferred using electrodes of same size. EWOD devices require specific patterning of electrodes for droplet actuation which can be a tedious procedure. The tolerances for such devices are quite low and hence accuracy required is extremely high. In DEP, the fabrication of electrodes for separation in general is comparatively simpler because an array of electrodes like EWOD or OEW is not essential. Thermo-capillary devices require special temperature gradient producing systems embedded into the microfluidic device which again calls for extreme intricacy and accuracy for perfect device functionality. Similarly for SAW devices, the IDT electrodes, for propagation of acoustic waves, require patterning on the device plane for actuation of droplets. Typically elements like platinum are used and handling them at such a small scale may turn out to be a herculean task. Due to the advent of IC technology device fabrication for EWOD and $\mathrm{OEW}$ is not a significant problem as is for other techniques. As regards of this index, all techniques stand on an almost equal footing.

The most important consideration for any potential application is the cost involved in development. As quite evident, silicon is the major component of many devices with other materials like glass, PDMS, plastics etc. Although, IC technology has become cheaper down the line, but due to the high level of intricate design required for microfluidic devices, economy remains a major concern. Apart from the fabrication cost, all techniques have other major costs involved regarding the materials used in the device. Electro-wetting devices incorporate dielectrics making it EWOD, the most significant component of Optofluidic devices is the photoconductive material and the laser/other optical source used for manipulation, and Dielectrophoretic devices require PDMS and other electrode materials. Thermo-capillary devices require temperature gradient devices at the micro-scale which are difficult to manufacture and also quite costly, SAW devices require RF oscillating materials with high modulus of elasticity like platinum which are not only quite expensive, but the other costs involved in incorporating the electrodes onto the devices are also a problem. Every technique has a backdrop on costs but on further individual analysis it may be possible to find the most economic choice. Opto-fluidic and SAW devices require a heavy investment in terms of required hardware. Thermo-capillary devices have a moderate range on this index depending on the temperature gradient source used. DEP and EWOD devices lie on the lower scale of this index.

It is worth noting that the cost index directly depends on the other two indices. There has to be a trade-off between the device functionality and the costs involved. Fabrication index more or less is in co-dependence with the economy factor. Depending on the specific purpose of application, the different indices may be given appropriate weightage in order to find the most optimum technique to be used for making the device. But for all intents and purposes, these factors are solely based on the premise that at some point Digital Microfluidics will see large scale manufacturing.

\section{Conclusion}

The paper gives a treatise on the techniques used in Digital Microfluidics. The complete analysis of the various techniques currently used for digitization of fluids, by numerous researchers is presented.

Performance Indices for selection criteria have been proposed for selecting the appropriate technique, given a specific application that is intended. It is worth noting that even though several advantages of using Digital Microfluidics have been evaluated and proposed over the period of years; this technology is yet to revolutionize the biomedical 
Citation: Sukhatme S, Agarwal A (2012) Digital Microfluidics: Techniques, Their Applications and Advantages. J Bioengineer \& Biomedical Sci S8:001. doi:10.4172/2155-9538.S8-001

market by entering mass production. Hence, many of the proposed ideas are based on the research point-of-view; however their selection should be based on the mass reproducibility of these devices.

\section{References}

1. Teh SY, Lin R, Hung LH, Lee AP (2008) Droplet microfluidics. Lab Chip 8: 198220 .

2. Fair RB (2007) Digital microfluidics: is a true lab-on-chip possible Microfluid Nanofluid 3: 245-281.

3. Stone HA Stroock AD, Ajdari A (2004) Engineering flows in small devices:Microfluidics toward alab-on-a-chip. Annu Rev Fluid Mech. 36: $381-$ 411.

4. Link DR, Grasland-Mongrain E, Duri A, Sarrazin F, Cheng Z, et al. (2006) Electric control of droplets in microfluidic devices. Angew Chem Int Ed Engl 45: 2556-2560.

5. Whitesides GM (2006) The origins and the future of microfluidics. Nature 442: 368-373.

6. Mugele F, Baret JC (2005) Electro-wetting: from basics to applications. J Phys Condens Matter 17: 705-774.

7. Pollack MG, Fair RB, Shenderov AD (2000) Electro-wetting based actuation of liquid droplets for microfluidic applications. Appl Phys Lett 77.

8. Fair RB, Khlystov A, Tina DT, Ivanov V, Randall DE, et al. (2007) Chemical and Biological Applications of Digital-Microfluidic Devices. IEEE Design \& Test of Computers 24: 10-24.

9. Ren H, Fair RB, Pollack MG (2004) Automated on-chip droplet dispensing with volume control by electro-wetting actuation and capacitance metering. Sensors and Actuators B 98: 319-327.

10. Abdelgawad M, Park P, Wheeler AR (2009) Optimization of device geometry in single-plate digital microfluidics. J Appl Phys 105

11. Sung KC, Moon H, Chang JK (2003) Creating, Transporting, Cutting, and Merging Liquid Droplets by Electro-wetting Based Actuation for Digital Microfluidic Circuits. J Microelectromech Syst 12: 70-80.

12. Clime L, Brassard D, Veres T (2010) Numerical modeling of Electro-wetting transport processes for digital microfluidics. Microfluidics Nanofluidics 8: 599608.

13. Abdelgawad M, Aaron RW (2009) The Digital Revolution: A New Paradigm for Microfluidics. Adv Mater 21: 920-925.

14. Flavie S, Karine L, Laurent P, Christophe G, Thomas B, et al. (2006) Experimental and Numerical Study of Droplets Hydrodynamics in Microchannels. AIChE Journal 52: 4061-4069.

15. Lee J, Moon H, Fowler J, Kim CJ, Schoellhammer T (2001) Addressable micro liquid handling by electric control of surface tension. In: Proc. of the IEEE 14th Intl. Conf. on MEMS

16. Gong J, Kim CJ (2008) All-electronic droplet generation on-chip with real-time feedback control for EWOD digital microfluidics. Lab Chip 8: 898-906.

17. Chang YH, Lee GB, Huang FC, Chen YY, Lin JL (2006) Integrated polymerase chain reaction chips utilizing digital microfluidics. Biomed Microdevices 8: 215225 .

18. Srinivasan V, Pamula VK, Fair RB (2004) An integrated digital microfluidic labon-a-chip for clinical diagnostics on human physiological fluids. Lab Chip 4 310-315

19. Peykov V, Quinn A, Ralston J (2000) Electro-wetting: a model for contact-angle saturation. Colloid Polym Sci 278: 789-793.

20. Ashkin A (2000) History of optical trapping and manipulation of small-neutral particle, atoms, and molecules. IEEE J Sel Top Quantum Electron 6: 841-856.

21. Gahagan KT, Swartzlander GA Jr (1996) Optical vortex trapping of particles. Opt Lett 21: 827-829.

22. Grier DG (2003) A revolution in optical manipulation. Nature 424: 810-816.
23. Chiou PY, Ohta AT, Wu MC (2005) Massively parallel manipulation of single cells and microparticles using optical images. Nature 436: 370-372.

24. Inui N (2007) Relationship between contact angle of liquid droplet and light beam position in opto-electro-wetting. Sensors and Actuators A 140: 123-130.

25. Chioua PY, Moonb H, Toshiyoshic H, Kimb CJ, Ming CW (2003) Light actuation of liquid by opto-electro-wetting. Sensors and Actuators A 104: 222-228.

26. Hwang H, Park JK (2011) Optoelectrofluidic platforms for chemistry and biology. Lab Chip 11: 33-47.

27. Chuang HS, Kumar A, Steven TW (2008) Open opto-electro-wetting droplet actuation. Appl. Phys. Lett. 93 064104: 1-4.

28. Pei SN, Valley JK, Neale SL, Jamshidi A, Hsu HY, et al. (2010) Light Actuated Digital Microfluidics for Large Scale Parallel Manipulation of Arbitrarily sized droplets. IEEE 23rd Intl. Conf. on Micro Electro Mechanical Systems (MEMS).

29. Chiou PY, Chang Z, Ming CW (2008) Droplet Manipulation With Light on Optoelectrowetting Device. Journal of Microelectromechanical Systems 17: 133-138

30. Pohl HA (1978) Dielectrophoresis: The behavior of neutral matter in nonuniform electric fields. Cambridge university press.

31. Gascoyne PR, Vykoukal J (2002) Particle separation by dielectrophoresis Electrophoresis 23: 1973-1983

32. Sung KC, Yuejun Z, Kim CJ (2007) Concentration and binary separation of micro particles for droplet-based digital microfluidics. Lab Chip 7: 490-498

33. Park SY, Kalim S, Callahan C, Teitell MA, Chiou EP (2009) A light-induced dielectrophoretic droplet manipulation platform. Lab Chip 9: 3228-3235.

34. Zhao Y, Yi UC, Cho SC (2007) Microparticle Concentration and Separation by Traveling-Wave Dielectrophoresis (twDEP) for Digital Microfluidics. J Microelectromech Syst 16: 1472-1481.

35. Timothy SS, Mark AB (1999) Thermo-capillary Pumping of Discrete Drops in Microfabricated Analysis Devices. AIChE Journal 45: 350-356.

36. Maria LC, Daniel RB, Charles NB, David MG (2008) Thermocapillary manipulation of droplets using holographic beam shaping: Microfluidic pin ball, Appl Phys Lett 93 0314107: 1-4.

37. Chen JZ, Troian SM, Darhuber AA, Wanger S (2005) Effect of contact angle hysteresis on thermocapillay droplet actuation. J Appl Phys 97.

38. Shi J, Mao X, Ahmed D, Colletti A, Huang TJ (2008) Focusing microparticles in a microfluidic channel with standing surface acoustic waves (SSAW). Lab Chip 8: 221-223.

39. Steven SS (2006) Fundamentals of BioMEMS and Medical Microdevices, SPIE Press.

40. Guttenberg Z, Muller H, Habermüller H, Geisbauer A, Pipper J, et al. (2005) Planar chip device for PCR and hybridization with surface acoustic wave pump. Lab Chip 5: 308-317.

41. Franke T, Braunmüller S, Schmid L, Wixforth A, Weitz DA (2010) Surface acoustic wave actuated cell sorting (SAWACS). Lab Chip 10: 789-794. 